\title{
Large surveys and determination of interstellar extinction
}

\author{
Oleg Yu. Malkov ${ }^{1,2}$ and Erkin Karimov ${ }^{2}$ \\ ${ }^{1}$ Center for Astronomical Data, Institute of Astronomy, Russian Academy of Sciences \\ (INASAN), Pyatnitskaya ul 48, RU-119017 Moscow, Russian Federation \\ email: malkov@inasan.ru \\ ${ }^{2}$ Sternberg Astronomical Institute, Moscow State University, \\ Universitetskij pr. 13, RU-119992 Moscow, Russian Federation \\ email: youju@mail.ru
}

The study of the spatial distribution of interstellar extinction, $A_{V}$, is important for many investigations of galactic and extragalactic objects. Three-dimensional (3D) extinction models have been produced using spectral and photometric stellar data, open cluster data, star counts, the Galactic dust distribution model.

The standard approach to construct a 3D extinction model has been to parcel out the sky in angular cells, each defined by boundaries in Galactic coordinates $(l, b)$. From the stars in each cell, the visual extinction $A_{V}(l, b)$ can then be obtained as a function of distance $A_{V}(l, b, r)$. The angular size of the cells has varied from study to study, although each cell was generally chosen to be large enough to contain a statistically significant number of calibration stars at different distances. Published 3D models, using spectral and photometric data, are based on 104-105 stars. Modern large surveys contain photometric ( 3 to 5 bands) data for $107-109$ stars. But to make that data useful for a 3D extinction model construction one needs to run a correct cross-identification of objects between surveys. Another problem is a lack of spectral data in photometric surveys.

Identification of objects requires the federation of multiple surveys obtained at different wavelengths and with different observational techniques. Such cross-matching of catalogs is currently laborious and time consuming. But using VO data access and crosscorrelation technologies a search for counterparts in a subset of different catalogues can be carried out in a few minutes. Particularly, information on interstellar extinction may be obtained from modern large photometric surveys data.

The goal of our paper is to design a procedure for construction of 3D interstellar extinction model, based on data from large surveys. To test the procedure we have selected a two-arc-minute area on the sky with $l=323, b=+6$. For further analysis the following multicolor surveys were chosen (photometric bands are given in brackets): DENIS $\left(J, K^{\prime}\right)$, 2MASS $(J, H, K s)$, USNO-B (SERC-J). Our two-arc-minute test area contains 134 objects cross-identified in all three surveys. For 36 of them all required photometry is available. We approximate our result by the relation: $A_{V}=0.01\left|\operatorname{cosec} 6^{\circ}\right|[1-$ $\left.\exp \left(-0.008 r\left|\sin 6^{\circ}\right|\right)\right]$. The uncertainty of $A_{V}$ is about $0 .{ }^{\mathrm{m}} 1$ depending primarily on the uncertainties of intrinsic colors. The relative error of the distance is about $25 \%$, depending primarily on the uncertainties of absolute magnitudes.

The proposed method has a number of advantages. One does not need for spectral type data and trigonometric parallaxes for calibration stars. One uses 104-106 times more stars than in 'traditional' models (it allows to choose angular cells on the sky small enough so that individual interstellar clouds can be resolved). 'On-line' model can be constructed to calculate $A_{V}(l, b, r)$ based on available data for a user defined area on the sky. When available, other multi-wavelength surveys like DPOSS, SDSS, UKIDSS can be incorporated using $\mathrm{VO}$ techniques. 